\title{
Electrical Measurements in GaN: Point Defects and Dislocations
}

\author{
David C. Look, Zhaoqiang Fang, and Laura Polenta ${ }^{1}$
}

Semiconductor Research Center, Wright State University, Dayton, OH 45435

${ }^{1}$ INFM and Dipartimento di Fisica, University of Bologna, I-40126 Bologna, Italy

\begin{abstract}
Defects can be conveniently categorized into three types: point, line, and areal. In $\mathrm{GaN}$, the important point defects are vacancies and interstitials; the line defects are threading dislocations; and the areal defects are stacking faults. We have used electron irradiation to produce point defects, and temperature-dependent Hall-effect (TDH) and deep level transient spectroscopy (DLTS) measurements to study them. The TDH investigation has identified two point defects, an 0.06-eV donor and a deep acceptor, thought to be the $\mathrm{N}$ vacancy and interstitial, respectively. The DLTS study has found two point-defect electron traps, at $0.06 \mathrm{eV}$ and $0.9 \mathrm{eV}$, respectively; the $0.06-\mathrm{eV}$ trap actually has two components, with different capture kinetics. With respect to line defects, the DLTS spectrum in as-grown GaN includes an $0.45-\mathrm{eV}$ electron trap, which has the characteristics of a dislocation, and the TDH measurements show that threading-edge dislocations are acceptor-like in n-type GaN. Finally, in samples grown by the hydride vapor phase technique, TDH measurements indicate a strongly n-type region at the $\mathrm{GaN} / \mathrm{Al}_{2} \mathrm{O}_{3}$ interface, which may be associated with stacking faults. All of the defects discussed above can have an influence on the dc and/or ac conductivity of GaN.
\end{abstract}

\section{INTRODUCTION}

Because GaN is a wide-bandgap semiconductor, it is commonly assumed that large quantities of point defects will be present, because of self-compensation affects. For example, the first GaN, grown some thirty years ago, was strongly n-type, and the natural assumption was that the relevant donors were associated with the $\mathrm{N}$ vacancy $\mathrm{V}_{\mathrm{N}}$ [1]. Only recently has this idea been shown not to be true, at least for the best, present-day GaN epitaxial layers [2]. In fact, accurate first-principles theoretical calculations indeed suggest a low concentration of $\mathrm{V}_{\mathrm{N}}$ centers in n-type GaN, but a much higher concentration of Ga vacancy $\mathrm{V}_{\mathrm{Ga}}$ defects [3]. However, the theoretical calculations 
assume equilibrium growth conditions, which may not be true, especially for the molecular-beam epitaxial (MBE) process. In fact, at the present time, MBE-grown GaN layers contain higher concentrations of donors, acceptors, and traps, than layers grown by metal organic chemical vapor deposition (MOCVD), or hydride vapor phase epitaxy

(HVPE). The question is whether those donors, etc., are associated with defects, or impurities, or both. With regard to line defects, i.e., dislocations, the situation is somewhat clearer. It is known that dislocations act as nonradiative recombination centers [4], and affect the performance of GaN-based light emitters [5]. More recently, it has been shown that they are also strong scattering centers, especially at the typical concentrations found in mismatched $\mathrm{GaN} / \mathrm{Al}_{2} \mathrm{O}_{3}$ layers [6]. Finally, there is evidence from deep-level transient spectroscopy (DLTS) experiments, that dislocations act as traps [7]. The $\mathrm{V}_{\mathrm{N}}$ center also is a trap [8].

Besides point defects and dislocations, there are two-dimensional defects, such as stacking faults, which we will classify as areal defects $[9,10]$. Recent theory suggests that stacking faults in GaN are not electrically active [10]; however, it is also known that the heavily-faulted interface region between $\mathrm{HVPE} \mathrm{GaN}$ and the $\mathrm{Al}_{2} \mathrm{O}_{3}$ substrate is strongly n-type [11], possibly due to donor-like defects, although impurities cannot be ruled out. Thus, point, line, and areal defects all may play important roles in determining the concentrations of donors, acceptors, traps, and recombination centers in GaN.

\section{LINE DEFECTS: DISLOCATIONS}

In GaN layers with high dislocation densities, the mobilities are usually low [12]. However, until recently, it was not clear whether or not dislocations had a direct or indirect effect on mobility. For example, one of the two layers shown in figure 1 has a much higher mobility than the other, and also a much lower dislocation density $\left[4 \times 10^{8}\right.$ $\mathrm{cm}^{-2}$ vs. $2 \times 10^{10} \mathrm{~cm}^{-2}$, respectively, as measured by transmission electron microscopy (TEM)]. But there are also other differences in the samples, which might be expected to have an even greater effect on the mobilities. To quantify the scattering effects of dislocations, we recently showed that the scattering rate for a line charge, such as a 


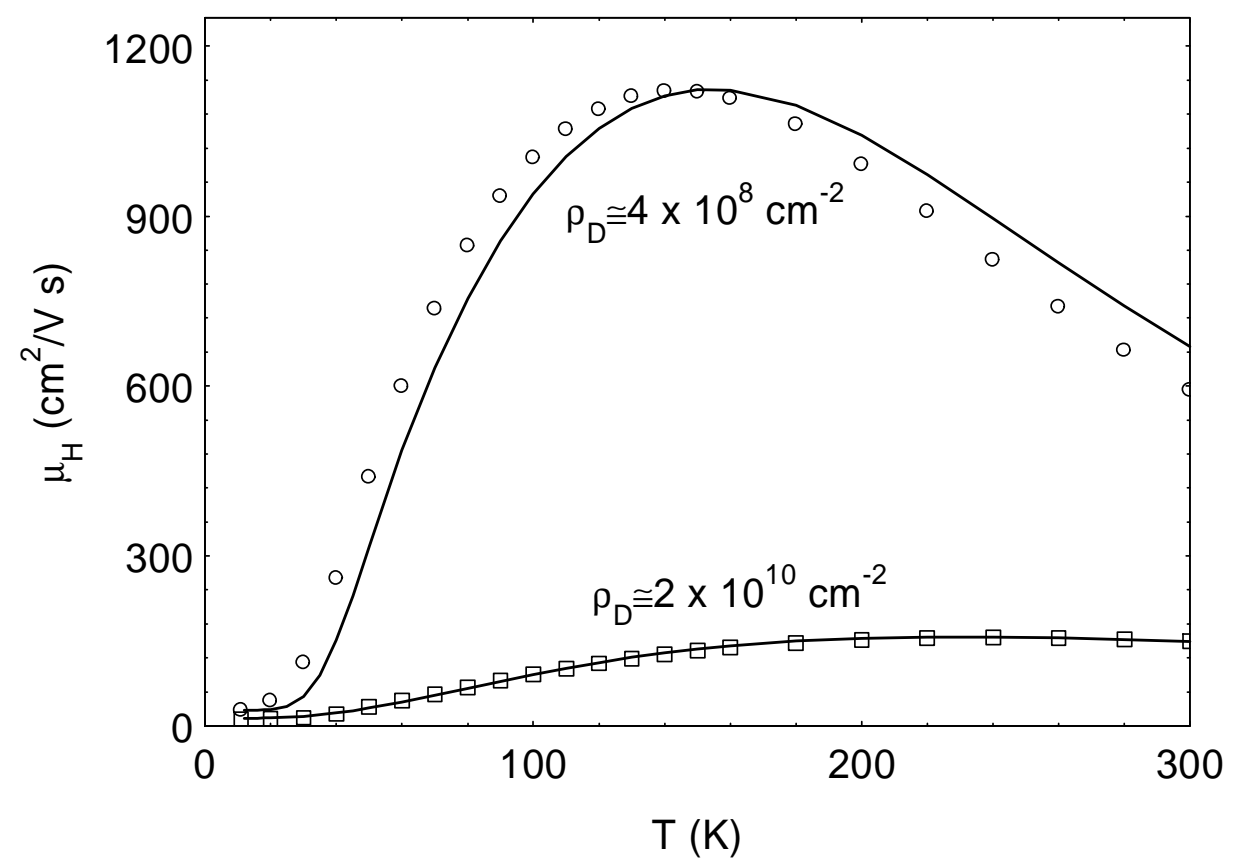

Figure 1. Hall mobility as a function of temperature for two MOCVD GaN samples with different dislocation densities $\left(\rho_{D}\right.$ 's). The solid lines are theoretical fits.

dislocation, is given by the inverse of the following relaxation time [6]:

$$
\tau_{\text {dis }}(k)=\frac{\hbar^{3} \varepsilon_{0}{ }^{2} c^{2}}{N_{\text {dis }} m^{*} f^{2} e^{4}} \frac{\left(1+4 \lambda^{2} k_{\perp}{ }^{2}\right)^{3 / 2}}{\lambda^{4}}
$$

where $\mathrm{c}$ is the c-lattice distance $(5.185 \AA), \mathrm{f} / \mathrm{c}$ is the linear charge density, $\lambda$ is a screening parameter, $\mathrm{k}_{\perp}$ is the wave vector in a direction perpendicular to the $\mathrm{c}$ axis, and the other symbols have their usual meanings. Since $\lambda \propto n^{1 / 2}$, it can be seen that dislocations are strongly screened by free electrons, and thus affect mobility more in the better (lower-n) samples. By adding the scattering rates of other important scattering mechanisms, and solving the Boltzmann transport equation to determine mobility, data sets such as those shown in figure 1 can be fitted, and parameters such as $\mathrm{N}_{\text {dis }}$ determined from the best fit [6]. The agreement shown in figure 1 is excellent, especially considering that the only 
fitting parameters, other than $\mathrm{N}_{\text {dis }}$, are the donor concentration $\mathrm{N}_{\mathrm{D}}$ and energy $\mathrm{E}_{\mathrm{D}}$. With an assumption of one negative charge per c-lattice distance $(\mathrm{f}=1)$, the fitted dislocation densities are $4.2 \times 10^{8}$ and $2.3 \times 10^{10} \mathrm{~cm}^{-2}$, respectively, in very good agreement with the TEM results. First-principles theory suggests that a likely configuration for the dislocation core in n-type $\mathrm{GaN}$ includes vacancies at the Ga sites [13]. It is expected that Ga vacancies could hold up to three negative charges per site ( $\mathrm{f}=3$ ); however, a theoretical analysis which includes electron-electron repulsion [14] leads to a value of about one charge per site $(\mathrm{f}=1)$, in agreement with the Hall results [6]. Note that such dislocations can strongly compensate the layer; e.g., $2 \times 10^{10}$ dislocations per $\mathrm{cm}^{2}$ will compensate $\left(2 \times 10^{10}\right) /\left(5.185 \times 10^{-8}\right)=4 \times 10^{17}$ electrons per $\mathrm{cm}^{3}$, a higher value than that found in some high-quality $\mathrm{GaN}$ layers these days. Dislocation densities less than $10^{8} \mathrm{~cm}^{-2}$ will have little effect on the mobility of present-day samples, but may become important again as impurity and defect levels are eventually reduced to the $10^{15}$ $\mathrm{cm}^{-3}$ level. It should also be pointed out that, besides being strong scattering centers, there is good evidence that dislocations can also act as recombination centers (RCs) [4] and traps [7]. With regard to the latter, consider trap $\mathrm{C} 1$, shown in the data of figure 2; pulsefilling studies verify that this trap has unusual capture kinetics, more suggestive of a line charge (dislocation) than a point charge. Note that trap and RC effects often begin to show up at far lower defect concentrations than those found to be important for compensation and scattering. Thus, efforts to rid GaN of threading dislocations should be vigorously pursued.

\section{AREAL DEFECTS: STACKING FAULTS}

Type I stacking faults (one violation of the stacking rule) in GaN have a small formation energy (10 meV per unit-cell area), and thus are expected to be abundant [10]. Indeed, they are quite common in $\mathrm{GaN}$, especially near the film/substrate interfaces [9]. In HVPE-grown GaN, especially, a region of about 2000- $\AA$ thickness near the $\mathrm{GaN} / \mathrm{Al}_{2} \mathrm{O}_{3}$ interface is heavily faulted, and, interestingly, this same region is strongly ntype, often with a sheet density of about $10^{15} \mathrm{~cm}^{-2}$ or greater [11]. There is some disagreement in the literature whether or not stacking faults (SFs) should have electronic levels in the band gap; one theoretical calculation finds a level at $\mathrm{E}_{\mathrm{V}}+0.13 \mathrm{eV}$ [15], 


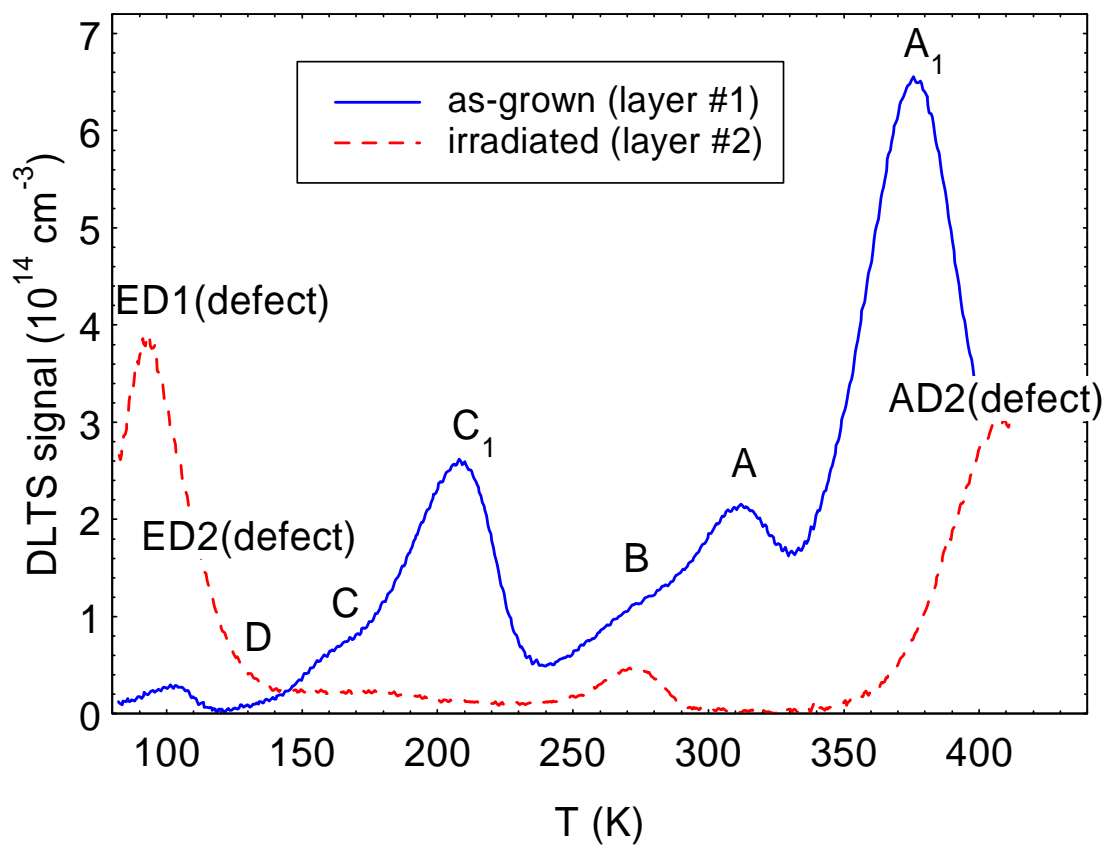

Figure 2. DLTS spectra for an as-grown MBE GaN layer (solid line), and an irradiated MOCVD GaN layer (dashed line).

while another finds no levels at all [10]. Neither of these scenarios can explain the highly degenerate n-type region, so that the origin of these shallow donors remains a mystery. It is possible that other donor-type defects, such as $\mathrm{N}$ vacancies, are present along with the $\mathrm{SFs}$, or there may be a high concentration of impurity donors, such as $\mathrm{O}_{\mathrm{N}}$. In any case, this interface region has a strong effect on the measured electrical properties, as seen in figure 3. In this figure, the dashed line represents the true (bulk) mobility, determined by analyzing the Hall data with a two-layer conduction model. The carrier concentration (not shown) is affected even more, and an analysis of the raw (uncorrected) concentration data, without accounting for the interface region, leads to very inaccurate donor energies [11]. Also, any devices fabricated on such layers could experience severe current shunting. The elimination of these interface donors, whether or not they result directly from defects, would be highly desirable. 


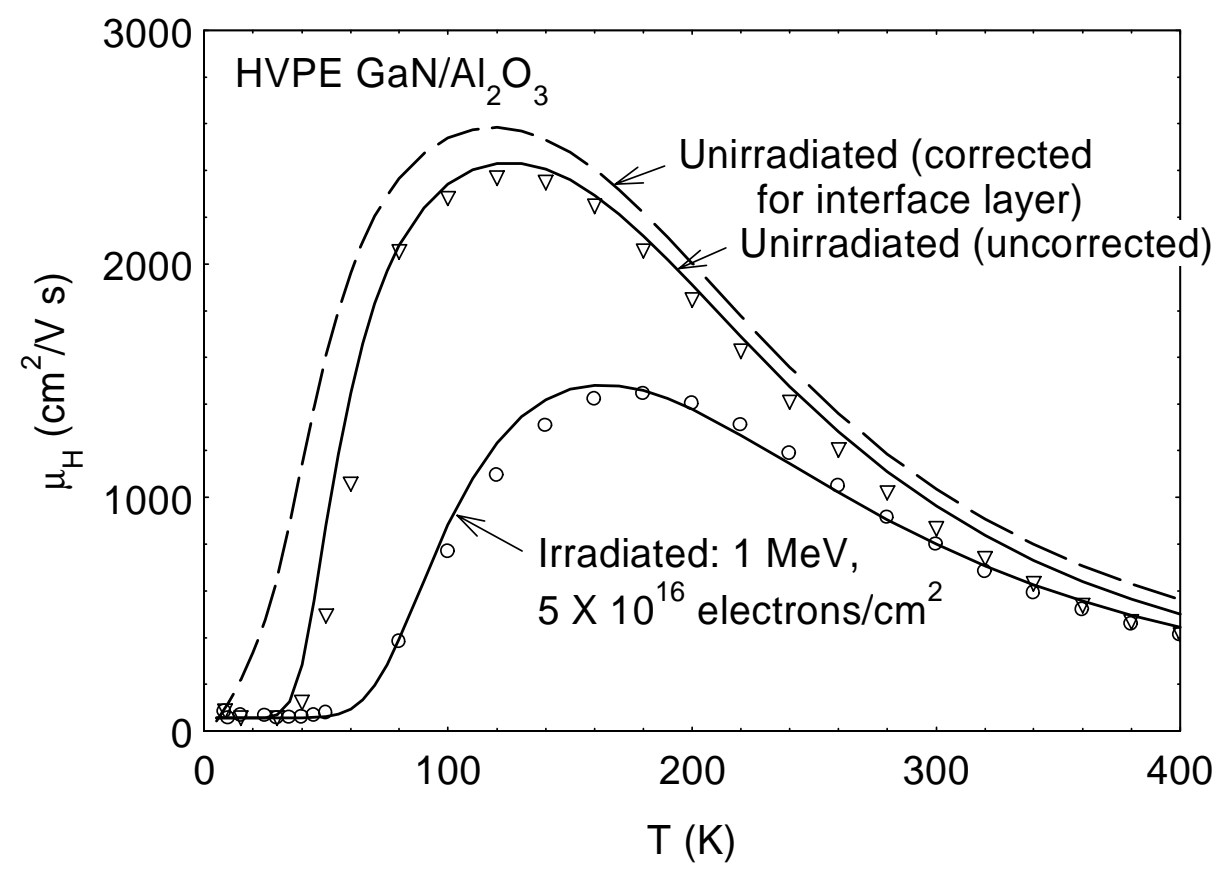

Figure 3. Hall mobility as a function of temperature for unirradiated and irradiated HVPE GaN layers. Also shown (dashed line) is the mobility of the unirradiated sample corrected for a degenerate interface layer.

\section{POINT DEFECTS: VACANCIES AND INTERSTITIALS}

Although point defects have longed been blamed for many of the undesirable properties of GaN materials, it is only in recent years that some of the true facts have become known. To be able to accurately investigate defects, it must be possible to produce them in a controlled manner, and this process can be carried out with a highenergy electron beam, which knocks out atoms, but does not create massive damage and large defect complexes [16]. Figure 3 shows the typical mobility degradation due to irradiation with $1-\mathrm{MeV}$ electrons to a fluence of $5 \times 10^{16}$ electrons per $\mathrm{cm}^{2}$. The sample here is a $60-\mu \mathrm{m}$-thick, $\mathrm{HVPE}, \mathrm{GaN} / \mathrm{Al}_{2} \mathrm{O}_{3}$ layer, which, before irradiation, exhibited a "world's record" mobility of $950 \mathrm{~cm}^{2} / \mathrm{V}$-s at room temperature. Theoretical fits of the $\mu$ vs.-T data (figure 3), and n-vs.-T data (not shown), indicate that each 1-MeV electron per $\mathrm{cm}^{2}$ produces about one $0.06-\mathrm{eV}$ donor, and one deeper acceptor per $\mathrm{cm}^{3}$, and the 
interpretation, from several considerations, is that the donor is the $\mathrm{N}$ vacancy, and the acceptor, the $\mathrm{N}$ interstitial [2]. An immediate conclusion from these assignments is that the residual donor in this material, and in most other state-of-the-art GaN layers, is not $\mathrm{V}_{\mathrm{N}}$, because the residual donor energy in such layers is typically only about $0.02 \mathrm{eV}$, not $0.06 \mathrm{eV}$. The usual impurity candidates suggested for residual donors are $\mathrm{O}_{\mathrm{N}}$ and $\mathrm{Si}_{\mathrm{Ga}}$, and, indeed, each of these would be expected to have an energy of about $0.02 \mathrm{eV}$ at the $10^{17}-\mathrm{cm}^{-3}$ level. Unfortunately, however, it is difficult to distinguish between these two impurities, because it is not easy at the present time to accurately determine concentrations of $\mathrm{O}$ and $\mathrm{Si}$ (or anything else) in the low $-10^{17}-\mathrm{cm}^{-3}$ range. For some samples having very high free-electron concentrations, say higher than mid-10 $18 \mathrm{~cm}^{-3}$, there is evidence that impurities cannot account for all of the donors; in such cases, $V_{N}$ donors may also be important, but verification is difficult because the donor-energy fingerprint (i.e., $0.06 \mathrm{eV}$ ) will be greatly modified by screening effects in highconcentration samples.

We now consider the use of DLTS to study point defects created by irradiation. The dashed line in figure 4 shows the change in the DLTS spectrum of a $4-\mu \mathrm{m}$-thick MOCVD $\mathrm{GaN} / \mathrm{Al}_{2} \mathrm{O}_{3}$ layer after a $1-\mathrm{MeV}$ electron irradiation to a fluence of $6 \times 10^{15} \mathrm{~cm}^{-2}$. The only trap produced by the irradiation (in this DLTS temperature range) is the one designated $\mathrm{E}$, with a peak near $115 \mathrm{~K}$. It turns out that trap $\mathrm{E}$ is produced by several types of irradiation, including electrons [8], protons [17], He ions [7], and sputtered Au atoms [18], and thus must be a rather common point defect. The usual Arrhenius analysis of $E$ gives energies ranging from 0.13 to $0.20 \mathrm{eV}$, depending on the type of irradiation $[7,8,17,18]$; however, very recently it has been shown that trap E, or at least the version produced by $1-\mathrm{MeV}$ electrons, really consists of two traps, ED1 and ED2, as shown in figure 5 [19]. (Note that trap D in this spectrum existed before the irradiation.) Both ED1 and ED2 have thermal energies of $0.06 \mathrm{eV}$, identical to that of the donor found from the TDH measurements; however, they have different capture cross sections, with that of ED2 being temperature dependent. It is likely that ED1 and ED2 are both related to $\mathrm{V}_{\mathrm{N}}$, but that, in fact, they are different complexes of $\mathrm{V}_{\mathrm{N}}$. For example, one of them could be $\mathrm{V}_{\mathrm{N}}-\mathrm{N}_{\mathrm{I}}$, and the other, $\mathrm{V}_{\mathrm{N}}-\mathrm{N}_{\mathrm{Ga}}-\mathrm{Ga}_{\mathrm{N}}-\mathrm{N}_{\mathrm{I}}$. That is, from a simple theoretical model, presented in reference 20 , each of these "chain" defect complexes could be produced by $1-\mathrm{MeV}$ 


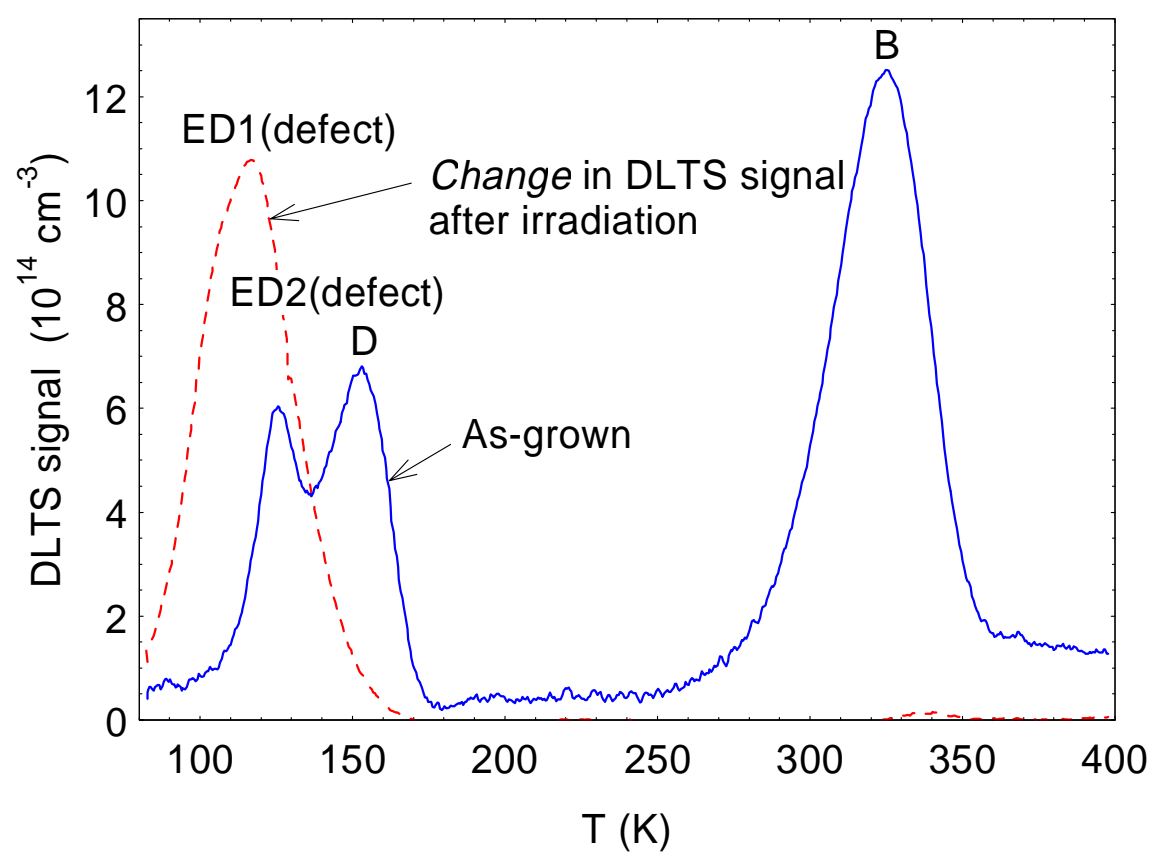

Figure 4. DLTS spectra for an as-grown MBE GaN layer (solid line), and an irradiated MOCVD GaN layer (dashed line). The spectrum for the irradiated sample actually represents only the change due to irradiation.

electrons. At a low enough irradiation energy, it should be possible to produce only the $\mathrm{V}_{\mathrm{N}}-\mathrm{N}_{\mathrm{I}}$ defect, if it is stable at room temperature, and the presence of only one defect would simplify the analysis. At present, an "exact" analysis, such as that shown in figure 5 , is absolutely essential to understand the important relationship between the irradiationproduced DLTS and TDH centers.

The practical importance of trap E becomes obvious when comparing with the DLTS spectrum of an as-grown $\mathrm{MBE} \mathrm{GaN} / \mathrm{Al}_{2} \mathrm{O}_{3}$ layer, also shown in figure 4. Note that the as-grown layer contains trap ED2, at a reasonably high concentration. There is independent evidence that trap ED2 (called $E_{l}$ in reference 21) is reduced by using higher $\mathrm{N}$ fluxes in the growth; this information is consistent with the $\mathrm{V}_{\mathrm{N}}$ nature of trap $\mathrm{E}$. 


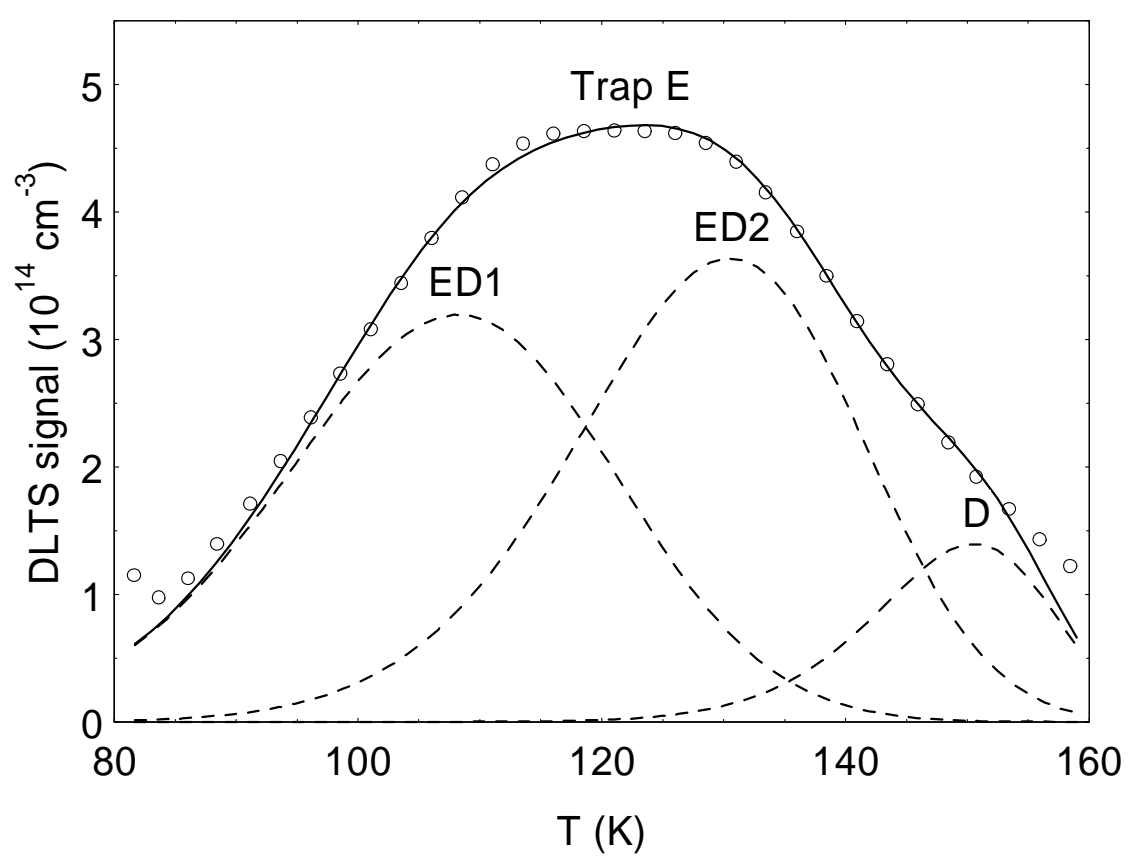

Figure 5. DLTS spectrum of Trap E. The solid line is the composite signal resulting from the three simulated traps shown as dashed lines.

By going to higher DLTS temperatures, another irradiation-induced trap appears, trap $\mathrm{AD} 2$, as shown in figure 2. The energy of trap AD2 is about $0.9 \mathrm{eV}$, close to that of a photoluminescence band which appears in electron-irradiated $\mathrm{GaN}$, and which has tentatively been assigned to a $\mathrm{Ga}_{\mathrm{I}} \mathrm{X}$ complex [22]. Interestingly, the as-grown MBE GaN layer shown in figure 2 displays trap $\mathrm{A}_{1}$, which also has an energy of about $0.9 \mathrm{eV}$, and may be related to the defect trap AD2. (Trap $A_{1}$ also appears in MOCVD and HVPE layers.) Traps ED2 and AD2 may be expected to influence GaN devices, because these traps can reach levels of $10^{15} \mathrm{~cm}^{-3}$ or higher in some samples.

Another point defect which has been studied in as-grown $\mathrm{GaN}$ is the $\mathrm{Ga}$ vacancy, $\mathrm{V}_{\mathrm{Ga}}$. Theory suggests that $\mathrm{V}_{\mathrm{Ga}}$ centers should be prevalent in n-type $\mathrm{GaN}$ [3], and indeed, they have been identified by positron annihilation experiments [23]. It is a mystery as to why the $\mathrm{V}_{\mathrm{Ga}}$ defect has not yet been found in irradiated $\mathrm{GaN}$, but the $\mathrm{V}_{\mathrm{Ga}}-\mathrm{Ga}_{\mathrm{I}}$ Frenkel 
pairs possibly recombine as soon as they are created, in n-type material, due to the strong coulomb attraction between $\mathrm{V}_{\mathrm{Ga}}{ }^{3-}$ and $\mathrm{Ga}_{\mathrm{I}}{ }^{+}$.

The results presented here show that point defects in GaN can be donors, acceptors, traps, and recombination centers, and that they exist in as-grown GaN layers. Although not specifically discussed in this paper, it is also important to note that defects will be produced in a radiation environment, such as that associated with the Van Allen belt. Future tasks include showing how point defects may affect particular types of devices, and then, how to get rid of them. This program is likely to engage the GaN community for some time to come.

\section{SUMMARY}

Three types of defects, point, line, and areal, have been found in n-type GaN, and can influence the electrical properties of the material. Line defects, namely threading dislocations, have been shown to act as acceptors, recombination centers, scattering centers, and possibly traps, while areal defects, stacking faults in this case, induce a highly conductive interface region in HVPE GaN/ $\mathrm{Al}_{2} \mathrm{O}_{3}$ layers. We have employed highenergy electron irradiation to create point defects, and have used TDH and DLTS to show that at least one of them is a donor, and that two of them are traps. We have also shown that very similar traps exist in as-grown GaN layers, and may have an effect on GaN devices.

\section{ACKNOWLEDGMENTS}

We wish to thank H. Morkoç and R. Molnar for samples; S. Keller for raw, Hall data; and J.W. Hemsky for electron irradiations. D.C.L. and Z-Q. F. were supported under U.S. Air Force Contract F33615-95-C-1619 and U.S. Navy Grant N00014-99-11067. LP was supported by INFM (Italian National Institute for Physics of Matter). Also, partial support was received from the Air Force Office of Scientific Research. Most of the work of D.C.L. was performed at the Air Force Research Laboratory, WrightPatterson Air Force Base, Ohio.

\section{REFERENCES}

1. H. P. Maruska and J.J. Tietjen, Appl. Phys. Lett. 15, 327 (1969). 
2. D.C. Look, D.C. Reynolds, J.W. Hemsky, J.R. Sizelove, R.L. Jones, and R.J. Molnar, Phys. Rev. Lett. 79, 2273 (1997).

3. J. Neugebauer and C. G. Van de Walle, Phys. Rev. B 50, 8067 (1994).

4. S. Evoy, H.G. Craighead, S. Keller, U.K. Mishra, and S.P. DenBaars, J. Vac. Sci.

Technol. B 17, 29 (1999).

5. T. Mukai, K. Takekawa, and S. Nakamura, Jpn. J. Appl. Phys. 37, L839 (1998).

6. D.C. Look and J.R. Sizelove, Phys. Rev. Lett. 82, 1237 (1999).

7. F.D. Auret, S.A. Goodman, F.K. Koschnick, J-M. Spaeth, B.Beaumont, and P.Gibart, Appl.Phys. Lett. 73, 3745 (1998).

8. Z-Q. Fang, J.W. Hemsky, D.C. Look, and M.P. Mack, Appl. Phys. Lett. 72, 448(1998).

9. L.T. Romano, B.S. Krusor, and R.J. Molnar, Appl. Phys. Lett. 71, 2283 (1997).

10. C. Stampfl and C.G. Van de Walle, Phys. Rev. B 57, R15052 (1998).

11. D.C. Look and R.J. Molnar, Appl. Phys. Lett. 70, 3377 (1997).

12. S. Keller, B.P. Keller, Y-F. Wu, B. Heying, D. Kapolnek, J. S. Speck, U. K. Mishra, and S. P. DenBaars, Appl. Phys. Lett. 68, 1525 (1996).

13. A.F. Wright and U. Grossner, Appl. Phys. Lett. 73, 2751 (1998).

14. K. Leung, A.F. Wright, and E.B. Stechel, Appl. Phys. Lett. 74, 2495 (1999).

15. Z.Z. Bandić, T.C. McGill, and Z. Ikonić, Phys. Rev. B 56, 3564 (1997).

16. F. Agullo-Lopez, C.R.A. Catlow, and P.D. Townsend, Point Defects in Materials, (Academic, New York, 1988).

17. F.D. Auret, S.A. Goodman, F.K. Koschnick, J-M. Spaeth, B.Beaumont, and P.Gibart, Appl.Phys. Lett. 74, 407 (1999).

18. F.D. Auret, S.A. Goodman, F.K. Koschnick, J-M. Spaeth, B.Beaumont, and P.Gibart, Appl.Phys. Lett. 74, 2173 (1999).

19. L. Polenta, Z-Q. Fang, and D.C. Look, 1999 (unpublished).

20. D.C. Look, J.W. Hemsky, and J.R. Sizelove, Phys. Rev. Lett. 82, 2552 (1999).

21. Z-Q. Fang, D.C. Look, W. Kim, Z. Fan, A. Botchkarev, and H. Morkoç, Appl. Phys. Lett. 72, 2277 (1999).

22. M. Linde, S.J. Uftring, G.D. Watkins, V. Härle, and F. Scholz, Phys. Rev. B 55,

R10177 (1997).

23. K. Saarinen, J. Nissilä, P. Hautojärvi, J. Likonen, T. Suski, I. Grzegory, B. Lucznik, and S. Porowski, Appl. Phys. Lett. 75, 2441 (1999). 